\title{
Artificial Inoculation of Bacterial Leaf Blight (BLB) Pathogen/ Inoculum on Different Entries of Cotton Crop under South Gujarat Region, India
}

\author{
Prashant B. Sandipan ${ }^{1 *}$, G.R. Bhanderi ${ }^{1}$, R.D. Patel ${ }^{1}$, R.K. Patel $^{2}$, \\ G.O. Faldu ${ }^{1}$ and M.C. Patel ${ }^{1}$ \\ ${ }^{1}$ Main Cotton Research Station (MCRS), Navsari Agricultural University (NAU), \\ Surat 395007 (Gujarat), India \\ ${ }^{2}$ Regional Cotton Research Station (RCRS), NAU, Bharuch (Gujarat), India \\ *Corresponding author
}

\begin{abstract}
A B S T R A C T
Keywords

Cotton, Bacterial

blight, Screening,

Entries, Artificial

inoculation

Article Info

Accepted:

04 September 2018

Available Online:

10 October 2018

Cotton is a very important commercial crop of India and to the world. Essentially used for fibre, fuel and edible oil and various other purposes. It is important because it is cash crop to the farmers. Out of 30 diseases known to occur in cotton crop from time to time, the bacterial blight is the most wide spread and destructive disease reported to cause yield losses of about 10 to 30 per cent. In this experiment different cultivars/ entries were screened against the bacterial leaf blight disease. Infester row of susceptible check LRA 5166 was grown alternating every four rows of the test entries. The maximum PDI of LRA 5166 was 20.0 PDI was observed. Artificial inoculation was made twice by preparing the spore suspension of BLB. Total 44 entries were screened through artificial inoculation, from these entries viz., GN Cot. 22, GISV 272, GSHV 180 and G. Cot.20 (LC) were moderately resistant against the bacterial leaf blight disease whereas, in case of Alternaria leaf spot disease, three entries viz., GISV 272, GSHV 180 and G. Cot. 20 (LC) were observed as resistant against the disease. Rests of the entries were free from the infection.

\section{Introduction}

Cotton plays an important role in sustaining the Indian economy as India is the world's second largest cotton producer (Khadi et al., 2009). According to Agrios (2005), the estimated $36.5 \%$ average of total losses includes $14.1 \%$ caused by diseases (fungi, bacteria and viruses), $10.2 \%$ by insects and $12.2 \%$ by weeds. Largest crop loss (14.1\%) is by phytopathogens alone. Among the phytopathogens, over 30 species of fungi can cause cotton plant infections (Farrell and

Johnson, 2005). The pathogen attacks host plants during all growth stages, infecting stems, leaves, bracts and bolls and causes seedling blight, black arm, angular leaf spot, and boll lesions (Verma, 1986). Symptoms show that lesions turn black as the age and increase in size. The affected leaves will have a torn appearance and premature defoliation occurs. Systemic infections follow the main veins of the leaf and appear as black streaks. Lesions associated with Bacterial Blight are generally darker in colour (Photograph 1). Yield losses due to bacterial blight range
\end{abstract}


between $1 \%$ and $27 \%$ depending on the cultivar and crop age (Mishra and Ashok Krishna 2001). Under natural bacterial blight infection, boll yield losses up to $35 \%$ have been reported (Sheo Raj and Verma 1988). Bacterial blight disease caused by Xanthomonas campestris pv. malvacearum (Smith) Dye, [which is the same as $X$. axonopodis pv. malvacearum (Smith) (Vauterin et al., 2000)] can be a serious disease in most Upland cotton (Gossypium hirsutum L.) growing areas of the world. Yield losses of 10 to $30 \%$ (Thaxton and El-Zik, 2001). Bacterial blight disease (BLB) of cotton affects the entire aerial parts of cotton plant i.e. necrosis of parenchymatous tissue in the local phase and blockage of xylem vessels in its systemic phase (Casson et al., 1977 and Sandipan et al., 2015). Control has been achieved through the use of resistant cultivars and/ or cultural methods, including aciddelinting seed, sanitary practices during ginning and processing, applying fungicides to seed and the destruction of diseased plant residues following tillage (Thaxton and ElZik, 2001). The purpose of the experiments was to determine the bacterial leaf blight reaction of cotton cultivars. Information may focus about their blight responses may be useful for further studies in incorporating blight resistance into future cotton cultivars/ entries.

\section{Materials and Methods}

The susceptible cultivar LRA - 5166 were sown after each four entry in this experiment by dibbling method with the following experimental details (Table 1). All the recommended agronomic practices were followed for raising the good crop. In each plot of each treatment randomly tag 5 plants and score 5 lower and 5 middle leaves of each plant in terms of 0-4 grade and work out PDI as mentioned below by using 0-4 scale as given by Sheoraj, 1988 and then these grades were converted into per cent disease incidence (PDI) by using the formula given by Wheeler, 1969 (Bacterial leaf blight and Alternaria leaf spot disease).

$$
\text { No. of infected plants }
$$

Disease incidence $(\%)=$

No. of leaves observed x Max. Grade

\section{Collection of the samples}

Samples of naturally infected with bacterial leaf blight of cotton plants were collected from Research farm from the Main Cotton Research Station (MCRS), Surat during Kharif, 2016. The infected leaf part of the diseased samples were carefully placed in polythene bags, properly tagged and brought to the laboratory. Samples were thoroughly washed with sterile distilled water. The proper/ appropriate amount of infected leave was collected for the spore suspension to prepare. The diseased bits (Infected cotton leaves) were given a cut with sharp sterilized blade. The bits were left for one minute to allow bacterial ooze to come out in water and adjusted around to $10^{7}$ colony forming units (cfu)/ ml. Approximately 15-20 days after inoculation, individual plants were graded for their disease reactions using the above formula.

\section{Results and Discussion}

The entries found disease free and resistant during previous year were tested this year under natural field and artificial condition against the different diseases. Infester row of susceptible check LRA 5166 was grown alternating every four rows of the test entries. The maximum PDI of LRA 5166 was 20.0 PDI was observed. Artificial inoculation was made twice by preparing the spore suspension of BLB. Total 44 entries were screened through artificial inoculation (Table 2), from these entries viz., GN Cot. 22, GISV 272, 
GSHV 180 and G. Cot.20 (LC) were GISV 272, GSHV 180 and G. Cot. 20 (LC) moderately resistant against the bacterial leaf were observed as resistant against the disease blight disease (Table 3) whereas, in case of (Table 4). Rests of the entries are free from the Alternaria leaf spot disease, three entries viz., infection.

\section{Photograph.1}

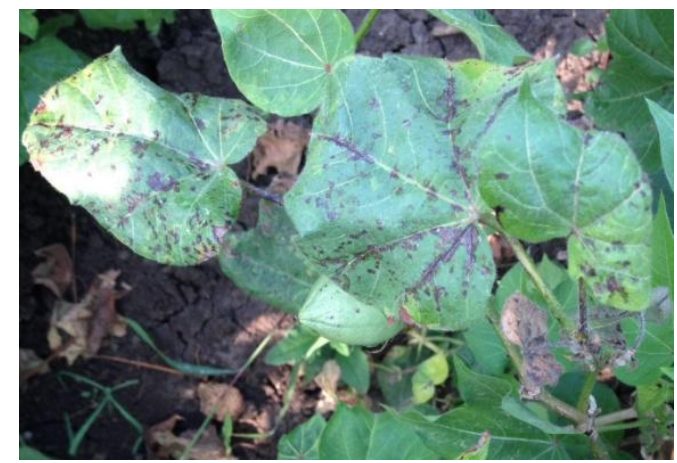

For, Bacterial leaf blight (BLB) disease

\begin{tabular}{|c|l|}
\hline Score & Description \\
\hline $\mathbf{0}$ & Immune, completely free from bacterial blight \\
\hline $\mathbf{1}$ & Highly resistant, infection $0-10 \%$ \\
\hline 2 & Moderately resistant, infection $11-20 \%$ \\
\hline 3 & Moderately susceptible, infection $21-40 \%$ \\
\hline 4 & Highly susceptible, infection more than $40 \%$ \\
\hline
\end{tabular}

For, Alternaria leaf spot (ALS) disease

\begin{tabular}{|c|}
\hline Score \\
\hline 0 \\
\hline 1 \\
\hline 2 \\
\hline 3 \\
\hline 4 \\
\hline
\end{tabular}

\section{Description}

No infection

Few $<2 \mathrm{~mm}$, scattered, brown spots, $<5 \& \%$ leaf area

Spots bigger, $3 \mathrm{~mm}$, not coalescing, brown and 6-20\% leaf area covered

Spots 3-5 mm, irregular in shape-coalescing,21-40\% leaf area covered

Spots coalescing to form bigger lesions, irregular->40 \% leaf area

Table.1

\begin{tabular}{|c|c|c|c|c|c|c|c|}
\hline $\begin{array}{l}\text { Location/ } \\
\text { Zone }\end{array}$ & $\begin{array}{l}\text { Variety/ } \\
\text { Entry }\end{array}$ & Rep. & $\begin{array}{c}\text { Plot size } \\
\text { (mtr) }\end{array}$ & $\begin{array}{l}\text { Spacing } \\
\text { (cm) }\end{array}$ & $\begin{array}{l}\text { Sowing } \\
\text { Date }\end{array}$ & $\begin{array}{c}\text { Fertilizer } \\
\text { NPK kg/ha }\end{array}$ & Irrigation \\
\hline $\begin{array}{c}\text { SG II } \\
\text { Surat } \\
\text { (Gujarat) }\end{array}$ & $\begin{array}{l}44+01 \\
\text { SC (LRA } \\
5166)\end{array}$ & 02 & $\begin{array}{c}1.2 \times 3.6 \\
\text { (Single } \\
\text { row) }\end{array}$ & $120 \times 45$ & 04.07 .16 & 240:40:00 & $\begin{array}{l}\text { As and } \\
\text { when } \\
\text { required }\end{array}$ \\
\hline
\end{tabular}


Table.2 Entries for confirmation and maintenance of disease resistant lines at MCRS, NAU, Surat (2016-17)

\begin{tabular}{|c|c|c|c|c|c|c|c|c|}
\hline \multirow[t]{2}{*}{ Sr. No. } & \multirow{2}{*}{\multicolumn{2}{|c|}{$\begin{array}{l}\text { Name of Entry } \\
\text { Code/Decode }\end{array}$}} & \multicolumn{3}{|c|}{ Bacterial leaf blight } & \multicolumn{3}{|c|}{ Alternaria leaf spot } \\
\hline & & & $\begin{array}{c}\text { Field } \\
\text { reaction }\end{array}$ & $\begin{array}{l}\text { Artificial } \\
\text { reaction }\end{array}$ & Reaction & $\begin{array}{c}\text { Field } \\
\text { reaction }\end{array}$ & $\begin{array}{l}\text { Artificial } \\
\text { reaction }\end{array}$ & Reaction \\
\hline \multicolumn{9}{|c|}{ Pr. Br. 02 (a) IET of G. hirsutum -Irrigated } \\
\hline 1 & \multicolumn{2}{|c|}{ GSHH 2729} & 0.0 & 0.0 & DF & 0.0 & 0.0 & DF \\
\hline 2 & \multicolumn{2}{|c|}{ GSHV 162} & 0.0 & 0.0 & DF & 0.0 & 0.0 & DF \\
\hline 3 & \multicolumn{2}{|c|}{ GN Cot. 22} & 0.0 & 5.5 & MR & 0.0 & 0.0 & DF \\
\hline \multicolumn{9}{|c|}{ Pr. Br. 03 (a) PVT of G. hirsutum-Irrigated } \\
\hline 4 & \multicolumn{2}{|c|}{ GSHV 172} & 0.0 & 0.0 & DF & 0.0 & 0.0 & DF \\
\hline 5 & \multicolumn{2}{|c|}{ GSHV 173} & 0.0 & 0.0 & DF & 0.0 & 0.0 & DF \\
\hline \multicolumn{9}{|c|}{ Pr. Br. 06 (b) Compact genotype } \\
\hline 6 & \multicolumn{2}{|c|}{ GISV 272} & 0.0 & 2.5 & MR & 0.0 & 3.0 & $\mathrm{R}$ \\
\hline 7 & \multicolumn{2}{|c|}{ GSHV 180} & 0.0 & 10.0 & MR & 0.0 & 2.5 & $\mathrm{R}$ \\
\hline 8 & \multicolumn{2}{|c|}{ G.Cot.20, LC } & 0.0 & 3.0 & MR & 0.0 & 2.0 & $\mathrm{R}$ \\
\hline \multicolumn{9}{|c|}{ Pr. Br. 22 a/b IET- G. arboreum } \\
\hline 9 & 881 & PA 793 & 0.0 & 0.0 & DF & 0.0 & 0.0 & DF \\
\hline 10 & 882 & ZC (AKA 7) & 0.0 & 0.0 & DF & 0.0 & 0.0 & DF \\
\hline 11 & 883 & PAIG 77 & 0.0 & 0.0 & DF & 0.0 & 0.0 & DF \\
\hline 12 & 884 & PA 781 & 0.0 & 0.0 & DF & 0.0 & 0.0 & DF \\
\hline 13 & 885 & PAIG 326 & 0.0 & 0.0 & DF & 0.0 & 0.0 & DF \\
\hline 14 & 886 & PA 827 & 0.0 & 0.0 & DF & 0.0 & 0.0 & DF \\
\hline 15 & 887 & PAIG 373 & 0.0 & 0.0 & DF & 0.0 & 0.0 & DF \\
\hline 16 & 888 & PAIG 368 & 0.0 & 0.0 & DF & 0.0 & 0.0 & DF \\
\hline 17 & 889 & PA 255 & 0.0 & 0.0 & DF & 0.0 & 0.0 & DF \\
\hline 18 & 890 & PA 778 & 0.0 & 0.0 & DF & 0.0 & 0.0 & DF \\
\hline 19 & 891 & PA 363 & 0.0 & 0.0 & DF & 0.0 & 0.0 & DF \\
\hline 20 & 892 & PA 760 & 0.0 & 0.0 & DF & 0.0 & 0.0 & DF \\
\hline 21 & 893 & PA 788 & 0.0 & 0.0 & DF & 0.0 & 0.0 & DF \\
\hline 22 & 894 & PA 08 & 0.0 & 0.0 & DF & 0.0 & 0.0 & DF \\
\hline 23 & 895 & G. Cot. 19 (LC) & 0.0 & 0.0 & DF & 0.0 & 0.0 & DF \\
\hline 24 & 896 & PA 796 & 0.0 & 0.0 & DF & 0.0 & 0.0 & DF \\
\hline 25 & 897 & PA 808 & 0.0 & 0.0 & DF & 0.0 & 0.0 & DF \\
\hline 26 & 898 & PA 741 & 0.0 & 0.0 & DF & 0.0 & 0.0 & DF \\
\hline \multicolumn{9}{|c|}{ Pr. Br. 32 b IET of G. herbaceum } \\
\hline 27 & 951 & RAHS 804 & 0.0 & 0.0 & DF & 0.0 & 0.0 & DF \\
\hline 28 & 952 & GShv $367 / 12$ & 0.0 & 0.0 & DF & 0.0 & 0.0 & DF \\
\hline 29 & 953 & GShv $371 / 12$ & 0.0 & 0.0 & DF & 0.0 & 0.0 & DF \\
\hline 30 & 954 & RAHS 801 & 0.0 & 0.0 & DF & 0.0 & 0.0 & DF \\
\hline 31 & 955 & GBhv 304 & 0.0 & 0.0 & DF & 0.0 & 0.0 & DF \\
\hline 32 & 956 & GBhv 307 & 0.0 & 0.0 & DF & 0.0 & 0.0 & DF \\
\hline 33 & 957 & ANGh 1601 & 0.0 & 0.0 & DF & 0.0 & 0.0 & DF \\
\hline 34 & 958 & GShv 362/12 & 0.0 & 0.0 & DF & 0.0 & 0.0 & DF \\
\hline 35 & 959 & GN Cot. 25 (LC) & 0.0 & 0.0 & DF & 0.0 & 0.0 & DF \\
\hline 36 & 960 & DWDh 1602 & 0.0 & 0.0 & DF & 0.0 & 0.0 & DF \\
\hline 37 & 961 & RAHS 802 & 0.0 & 0.0 & DF & 0.0 & 0.0 & DF \\
\hline 38 & 962 & ANGh 1602 & 0.0 & 0.0 & DF & 0.0 & 0.0 & DF \\
\hline 39 & 963 & RAHS 803 & 0.0 & 0.0 & DF & 0.0 & 0.0 & DF \\
\hline 40 & 964 & GShv 385/12 & 0.0 & 0.0 & DF & 0.0 & 0.0 & DF \\
\hline 41 & 965 & ZC (G.Cot.23) & 0.0 & 0.0 & DF & 0.0 & 0.0 & DF \\
\hline 42 & 966 & GBhv 302 & 0.0 & 0.0 & DF & 0.0 & 0.0 & DF \\
\hline 43 & 967 & DWDh 1601 & 0.0 & 0.0 & DF & 0.0 & 0.0 & DF \\
\hline 44 & 968 & GBhv 305 & 0.0 & 0.0 & DF & 0.0 & 0.0 & DF \\
\hline \multicolumn{3}{|c|}{ LRA 5166 (SC) } & 20.0 & 3 & MS & 0.0 & 0.0 & DF \\
\hline
\end{tabular}


Table.3 Reaction against the Bacterial leaf blight disease at MCRS, NAU, Surat (2016-17)

\begin{tabular}{|c|c|l|l|c|}
\hline Sr. No. & Grade & \multicolumn{1}{|c|}{ Reaction } & \multicolumn{1}{|c|}{ Total/ Number of entry } & No. of entries \\
\hline 1 & 0 & Disease free & Rest of all entries & 40 \\
\hline 2 & 1 & Resistant & \multicolumn{1}{|c}{-} & 0 \\
\hline 3 & 2 & Moderate resistant & $\begin{array}{l}\text { GN Cot. 22, GISV 272, GSHV 180, } \\
\text { G.Cot.20, LC }\end{array}$ & 4 \\
\hline 4 & 3 & Moderate susceptible & \multicolumn{1}{c}{0} \\
\hline
\end{tabular}

Table.4 Reaction against the Alternaria leaf spot disease at MCRS, NAU, Surat (2016-17)

\begin{tabular}{|c|c|l|l|c|}
\hline Sr. No. & Grade & \multicolumn{1}{|c|}{ Reaction } & \multicolumn{1}{|c|}{ Total/ Number of entry } & No. of entries \\
\hline $\mathbf{1}$ & $\mathbf{0}$ & Disease free & Rest of all entries & 41 \\
\hline $\mathbf{2}$ & 1 & Resistant & GISV 272, GSHV 180, G.Cot.20, LC & 3 \\
\hline $\mathbf{3}$ & 2 & Moderate resistant & - & 0 \\
\hline 4 & 3 & Moderate susceptible & - & 0 \\
\hline
\end{tabular}

\section{Acknowledgement}

Author is extremely appreciative to Main Cotton Research Station (MCRS), Surat (Gujarat) for providing the requisite facility and other required necessary arrangements for conducting the experiment.

\section{References}

Agrios, G.N. 2005 - Plant Pathology. 5th ed. Elsevier Academic Press, San Diego, California. pp. 3-15.

Casson, E. T., P. E. Richardson, L. A. Brinkerhoff and R. K. Gholson. 1977. Histopathology of immune and susceptible cotton cultivars inoculated with Xanthomonas campestris pv. malvacearum. Phytopathology, 67: 195196.

Farrell, T. and Johnson, A. 2005. Cotton pest management guide2005-06. NSW Department of Primary Industries; Cotton Catchment Communities CRC, Australia.

Khadi, B.M., Santhy, V. and Yadav, M.S. 2009. Cotton: An Introduction. In: Zehr U (ed) Biotechnology in Agriculture and Forestry. Vol.65 CottonBiotechnological Advances. SpringerVerlag Berlin Heidelberg, pp. 1-2.

Mishra S.P., Ashok Krishna (2001): Assessment of yield losses due to bacterial blight of cotton. Journal of Mycology and Plant Pathology, 31: 232-233.

Sandipan B. Prashant, Desai, H. R. and Solanki, B. G. 2015. Role of environmental factors on the bacterial blight (BLB) disease of cotton caused by Xanthomonas campestris pv. malvacearum under South Gujarat condition. The Bioscan 10 (4): 16411644.

Sheo Raj, Verma J.P. (1988): Diseases of cotton in India and their management. Review of Tropical Plant Pathology, 5: 207-254.

Thaxton, P.M., and K.M. El-Zik. 2001. Bacterial blight. p. 34-35. In T.L. Kirkpatrick and C.S. Rothrock (ed.) Compendium of Cotton Diseases. 2nd ed. Am. Phytopathol. Soc., St. Paul, $\mathrm{MN}$.

Vauterin, L., J. Rademaker, and J. Swings. 2000. Synopsis of the taxonomy of the 
genus Xanthomonas. Phytopathology 90:677-682.

Verma, J. P. (1986): Bacterial Blight of Cotton. CRC Press, Boca Raton: 278.
Wheeler, B. E. J. 1969. An Introduction to Plant Disease. John Willey and Sons, London, pages: 374 .

\section{How to cite this article:}

Prashant B. Sandipan, G.R. Bhanderi, R.D. Patel, R.K. Patel, G.O. Faldu and Patel, M.C. 2018. Artificial Inoculation of Bacterial Leaf Blight (BLB) Pathogen/ Inoculum on Different Entries of Cotton Crop under South Gujarat Region, India. Int.J.Curr.Microbiol.App.Sci. 7(10): 19-24. doi: https://doi.org/10.20546/ijcmas.2018.710.003 